\title{
IGUALDAD DE POSICIONES PARA LAS JUVENTUDES RURALES: EDUCACIÓN Y TECNOLOGÍA EN LA PROVINCIA DE SALta (ARgentina)
}

\author{
MARÍA Rosa Chachagua* \\ Universidad Nacional de Salta, CIITED-CONICET, Argentina \\ mariach208@gmail.com
}

Recibido: 15/12/2019 Aceptado: 17/2/2020

doi: $10.26439 /$ contratexto2020.n033.4784

Resumen. Anhelar una sociedad más igualitaria implica pensar en políticas públicas emancipadoras que se puedan aplicar en todos los ámbitos poblacionales, especialmente en la ruralidad. La educación es una de las herramientas fundamentales para el desarrollo de un país, por lo que su acceso es un derecho prioritario en todos los niveles (Gentili, 2009). Y este derecho abarca la posibilidad de ingresar al sistema educativo, estudiar, apropiarse, crecer, progresar y, finalmente, egresar para soñar con un futuro mejor. Este trabajo tiene como principal objetivo analizar las diferentes dimensiones de la desigualdad: socioeconómica, digital y de género, en el ámbito rural. En ese sentido, se discute la idea de igualdad de posiciones, planteada por Dubet (2011), tomando como casuística a los jóvenes que asisten a la Escuela Secundaria Rural Mediada por Tecnologías (ERMT) en la provincia de Salta (Chachagua, 2019). Otro de los objetivos es indagar qué sucede con la noción de juventudes en este ámbito, y para eso se pone en diálogo las distintas perspectivas de este concepto anclándolo en un territorio específico. Por último, a partir de la experiencia del trabajo de campo, se plantean propuestas de construcción colectiva vinculadas a la educación y la tecnología en ámbitos rurales. Propuestas que surgen de las voces de los propios actores (estudiantes, docentes, trabajadores), quienes se imaginan en escenarios futuros más igualitarios y con mayores (y mejores) posibilidades para todos.

Palabras clave: juventudes / tecnologías / ruralidad / políticas públicas / igualdad

* Doctora en Comunicación por la Universidad Nacional de La Plata, Argentina (https://orcid.org/0000-00018566-6175). 


\section{EQUAL POSITIONS FOR RURAL YOUTH: EDUCATION AND TECHNOLOGY IN THE PROVINCE OF SALTA (ARGENTINA)}

Abstract. Longing for a more equal society involves thinking about emancipatory public policies that can be applied in all population areas, especially in rural areas. Education is one of the fundamental tools for the development of a country, which means that access is a priority right at all levels (Gentili, 2009). Moreover, this right includes the possibility of entering into the educational system (studying, appropriating, growing, progressing and finally graduating) to dream of a better future. Our main objective is to analyze the different dimensions of inequality-socioeconomic, digital and genderin rural areas. In this sense, the idea of "equal positions" raised by Dubet (2011) is discussed, taking as study population the young people who attend the TechnologyMediated Rural Secondary School (hereinafter referred to as ERMT) located in the province of Salta (Chachagua, 2019). Another objective is to find out what happens to the notion of youth in this area. To that end, the different perspectives of this concept are discussed, by anchoring such concept in a specific territory. Finally, we have taken fieldwork experience to suggest collective construction proposals linked to education and technology in rural areas: proposals that arise from the voices of the actors (students, teachers, workers) who imagine themselves in more equal future scenarios with greater (and better) possibilities for all.

Keywords: youth / technologies / rurality / public policies / equality 


\section{POSIÇÕES IGUAIS PARA A JUVENTUDE RURAL: EDUCAÇ̃̃O E TECNOLOGÍA NA PROVÍNCIA DE SALTA (ARGENTINA)}

Resumo. 0 desejo de uma sociedade mais igualitária implica pensar em políticas públicas emancipatórias que possam ser aplicadas em todas as áreas da população, especialmente nas áreas rurais. A educação é uma das ferramentas fundamentais para o desenvolvimento de um país, o que implica que o acesso é uma prioridade, em todos os níveis (Gentili, 2009). E esse direito inclui a possibilidade de entrar no sistema educacional, estudar, se apropriar, crescer, progredir e finalmente se formar para sonhar com um futuro melhor. 0 principal objetivo deste trabalho é analisar as diferentes dimensões da desigualdade: socioeconômica, digital e de gênero nas áreas rurais. Nesse sentido, é discutida a idéia de igualdade de posições, levantada por Dubet (2011), tomando como estudo de caso os jovens que frequentam a Escola Secundária Rural Mediada por Tecnologias (ERMT) na província de Salta (Chachagua, 2019). Outro objetivo é investigar o que acontece com a noção de juventude nessa área, para que as diferentes perspectivas desse conceito sejam colocadas em diálogo, ancorando-o em um território específico. Por fim, levamos a experiência do trabalho de campo, para pensar em propostas de construção coletiva ligadas à educação e tecnologia nas áreas rurais. Propostas que surgem das vozes dos próprios atores (estudantes, professores, trabalhadores) imaginando em cenários futuros mais igualitários e com maiores (e melhores) possibilidades para todos.

Palavras-chave: juventude / tecnologias / ruralidade / políticas públicas / igualdade 


\section{INTRODUCCIÓN}

¿A qué nos referimos cuando hablamos de igualdad/desigualdad? ¿Qué horizontes de igualdad anhelamos? El principal objetivo de este trabajo es analizar las diferentes dimensiones de la desigualdad (socioeconómica, digital y de género) en el ámbito rural. Para ello se discute la idea de igualdad de posiciones, planteada por Dubet (2011), sobre la base de la casuística de los jóvenes, hombres y mujeres ${ }^{1}$, que asisten a la Escuela Secundaria Rural Mediada por Tecnologías (ERMT) en la provincia de Salta (Chachagua, 2019). Asimismo, se busca indagar qué sucede con la noción de juventudes en este ámbito, y para eso se pone en diálogo diferentes perspectivas de este concepto anclándolo en un territorio específico.

El punto de partida es la escuela pública. Allí es a donde llegaron las computadoras del Programa Conectar Igualdad (PCl) y donde se desarrolla la modalidad de educación mediada por TIC (tecnologías de la información y la comunicación). Por tanto, se constituye en la puerta de ingreso a las juventudes rurales y sus experiencias.

A lo largo del artículo se presentan diferentes testimonios de los jóvenes entrevistados en la sede de Santa Teresa, ubicada en el departamento de Anta en la provincia de Salta, para ilustrar la realidad que se vive en esa zona. A partir de la experiencia del trabajo de campo, se plantean propuestas de construcción colectivas, que surgen de las voces de los propios actores (estudiantes, docentes, trabajadores), quienes se imaginan en escenarios futuros más igualitarios y con mayores (y mejores) posibilidades para todos.

En primer lugar, se expone la metodología con la que se abordó la investigación. El apartado siguiente da cuenta de las nociones teóricas centrales: desigualdad, igualdad de posiciones y brecha digital; luego se describe el contexto salteño y se analiza cómo la desigualdad es persistente en esta provincia en sus diferentes dimensiones. A continuación, se discute la concepción de juventudes, su vínculo con la ruralidad, sus sueños y anhelos; y la desigualdad de género que se profundiza en estos ámbitos. Finalmente, se proponen ideas para mejorar las políticas públicas vinculadas a la tecnología y a la educación.

\section{METODOLOGÍA}

La metodología utilizada es mixta, es decir, combina los aspectos cualitativo y cuantitativo. Se aplicaron las siguientes técnicas de recolección de datos: observación en las

1 Por cuestiones de redacción y de uso del lenguaje, en adelante la frase "los jóvenes" se refiere a los hombres y mujeres cuyos casos se desarrollan en este artículo. 
aulas, encuestas a modo de exploración y entrevistas en profundidad a estudiantes y docentes de la escuela rural.

El trabajo de campo se llevó a cabo en diferentes etapas. Luego del primer acercamiento, se dialogó y entrevistó a la directora y a una trabajadora de la institución, para contar con la primera información de índole exploratoria. Así se acordaron intereses y objetivos para avanzar con la investigación. Posteriormente, se hicieron observaciones indirectas en la sede central, cuyas anotaciones se tomaron en un diario de campo.

A partir de la información que se fue recogiendo, se plantearon las primeras entrevistas a los docentes de la institución. Después, se procedió con las visitas a la sede de la escuela rural y se realizaron instancias de observación en el salón de clases. Más adelante, se efectuaron las encuestas a los estudiantes y, finalmente, las entrevistas a los estudiantes y los trabajadores. Cabe aclarar que en las visitas a la sede participó también el equipo directivo de la escuela, docentes y trabajadores de Unicef, ya que por la ubicación geográfica es imposible llegar por cuenta propia si no se cuenta con un vehículo personal apto para las condiciones del lugar.

Cuando decimos que esta investigación cuenta con aspectos cuantitativos, nos referimos a que tomamos los siguientes datos de referencia para la investigación:

- Censo Nacional 2010 (INDEC, 2010)

- Estadísticas oficiales del Instituto Nacional de Estadística y Censos (INDEC, 2010)

- Informe 2019: Escuelas Secundarias Rurales Mediadas por TIC, del Relevamiento Unicef (Fondo de las Naciones Unidas para la Infancia)

- Encuestas de autoría propia a los estudiantes de la ERMT

Y cuando nos referimos a los aspectos cualitativos, nos centramos principalmente en las narrativas de los estudiantes y docentes, obtenidas mediante las entrevistas que se aplicaron en diferentes momentos de la investigación.

La información recogida con las diferentes herramientas mencionadas fue analizada con un marco teórico anclado en los estudios culturales de la comunicación. Se estructura con base en tres tópicos y preocupaciones principales: desigualdades, juventudes y tecnologías. A partir de este cruce, fue posible estudiar las dinámicas y características de la ERMT. 


\section{CONTEXTO: EDUCACIÓN Y TECNOLOGÍA EN SALTA}

Unicef promueve un modelo educativo innovador en diferentes provincias de Argentina, denominado Escuela Rural Mediada por TIC (ERMT). En Salta se estableció en el año 2013, inicialmente, como un convenio cooperativo junto al Ministerio de Educación del Gobierno de la Provincia de Salta y el Programa Conectar Igualdad².

Su objetivo es garantizar el derecho de todos los jóvenes que viven en parajes rurales dispersos a acceder a la educación media y desarrollar competencias tecnológicas. Esta modalidad tiene una sede central, ubicada en un centro urbano de la ciudad de Salta Capital, a la que asisten todos los días los docentes; allí planifican y diseñan las clases que son compartidas con los estudiantes, que viven en distintas zonas rurales, a través de una plataforma educativa. Por otra parte, se han instalado sedes rurales, a donde asisten diariamente los estudiantes junto con un docente tutor, que coordina y gestiona el desarrollo de clases y la comunicación con los docentes que se encuentran en la sede central.

Existen catorce (14) sedes rurales en toda la provincia de Salta, Argentina (véase el mapa de la figura 1). Funcionan en los edificios de la escuela primaria de cada zona, ya que no cuentan con instituciones propias. En este sentido, Unicef fue un actor clave para el reacondicionamiento de esas edificaciones, pues, junto al gobierno provincial, se encargó de la conectividad y la instalación de los equipos necesarios. De igual manera, se logró una integración entre los niveles de educación primaria y secundaria, que no solo comparten las instalaciones, sino también la logística y demás actividades. Las sedes cuentan con un albergue donde muchos estudiantes se quedan a dormir durante la semana, y los viernes regresan a sus puestos ${ }^{3}$. En las zonas rurales salteñas, los puestos están muy alejados entre sí y, a su vez, entre ellos y la escuela. Por tal motivo, muchas veces los estudiantes y docentes deciden quedarse en la escuela, donde usan en común el comedor, la biblioteca, los baños y el conjunto de las instalaciones escolares. También desayunan, almuerzan, meriendan y cenan tanto estudiantes como docentes (muchos pertenecen a otra localidad/pueblo) de ambos niveles educativos.

2 Conectar Igualdad fue una de las políticas modificadas por el expresidente Mauricio Macri, la cual de ser una política pública del Estado Nacional se convirtió en una política TIC del Programa Educ. ar. Había transcurrido mucho tiempo sin que se distribuyeran computadoras, por lo que Unicef tuvo que hacerse cargo del equipamiento para los estudiantes rurales (Chachagua, 2019). Entonces no solo se generó un cambio de dependencia, sino la eliminación del presupuesto propio y el despido de cientos de trabajadores. Así dejó de ser una política pública de inclusión digital autónoma para volverse un programa de este portal educativo.

3 Los puestos son la denominación que reciben los hogares de estos estudiantes y sus familias. Tal categoría proviene del uso extendido en el ámbito rural argentino para referirse a cada una de las partes en las que se divide una finca para cuidar la hacienda, y a la vivienda que habita su responsable. 


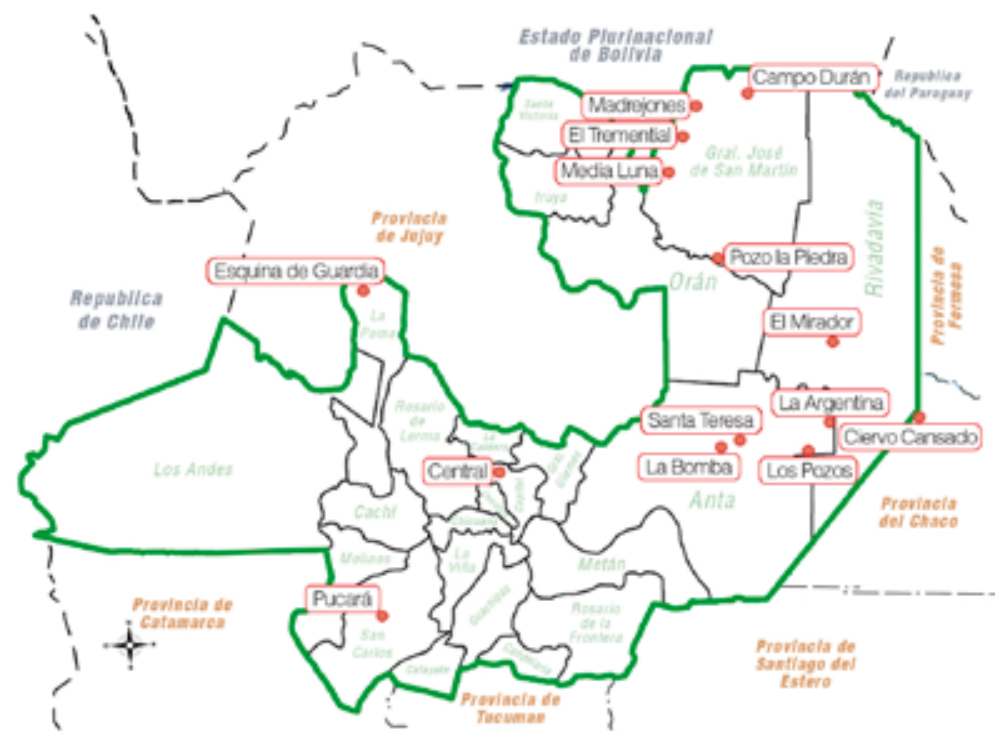

Figura 1. Mapa de Salta y sedes rurales

Elaboración propia

En este trabajo analizamos la sede de Santa Teresa, que está ubicada a 60 kilómetros de la localidad de Las Lajitas. Para llegar ahí es preciso contar con un vehículo propio, preferiblemente una camioneta, ya que el camino no es asfaltado y es muy complicado mantener el equilibrio por la cantidad de pozos existentes. Más aún, cuando llueve, estos terrenos se inundan y es imposible llegar a las escuelas. El traslado desde Las Lajitas a Santa Teresa demora un poco más de dos horas por lo difícil que es transitar ese camino.

En esta zona, el agua no es potable y tampoco se tiene acceso a luz eléctrica, lo que se debe a la ubicación geográfica, así como a la falta de inversiones de los diferentes gobiernos, tanto locales como provinciales. En cambio, cuentan con paneles solares ${ }^{4} y$ un generador eléctrico, que produce luz y agua para cada institución. Al carecer de luz eléctrica, se hace aún más difícil la conectividad, siendo este uno de los obstáculos más frecuentes para acceder a la educación ${ }^{5}$.

4 Un panel solar es un dispositivo que capta la energía del sol para su aprovechamiento. En la escuela tienen colectores solares, utilizados para producir agua caliente, y paneles fotovoltaicos, que generan electricidad mediante energía solar fotovoltaica.

5 En sus inicios, todas las sedes tenían antenas propias de ARSAT, mediante las cuales se podían conectar a internet. Sin embargo, por decisiones políticas establecidas por el expresidente Mauricio Macri, muchas empresas estatales y políticas de comunicación fueron modificadas, por lo que las sedes tuvieron que buscar otras opciones de conexión privadas. 
Durante la fase de exploración, una de las profesoras, que se encuentra en la sede central de la ciudad de Salta, comenta que esta modalidad "es una experiencia maravillosa, porque transforma la vida de los jóvenes en espacios alejados de lo urbano"; asimismo, resalta que "es un desafío constante, diariamente" (Luciana', docente, 47 años, entrevista realizada en abril del 2017). En la sede de Santa Teresa, Marian (14 años, estudiante de la ERMT, entrevista realizada en mayo del 2017) piensa que es una experiencia fantástica, pues "de otra manera no podríamos estudiar y nos tendríamos que dedicar a otra cosa; esta oportunidad que tenemos es muy valorable y no podemos desaprovecharla". Por su parte, Celeste (42 años, profesora de la sede central, entrevista realizada en abril del 2017) sostiene que este nuevo modelo de escuela posibilita que los jóvenes estudien, pero encuentra como principal desventaja los problemas de conexión. "Eso produce muchas fallas en la comunicación entre estudiantes, docentes y coordinadores".

\section{MARCO TEÓRICO: ¿QUÉ HORIZONTES DE IGUALDAD ANHELAMOS?}

Cuando se dice que América Latina es la región más desigual, se refiere a que es la más inequitativa del planeta. Sin embargo, no la más pobre del mundo en términos de disparidad de ingresos, si se la compara con algunos países en África, por ejemplo, que presentan mayores niveles de pobreza. Latinoamérica es el continente más desigual porque expresa los mayores contrastes entre las élites, que han disfrutado de las ventajas de la modernización, y la mayoría de la población, que ha vivido en condiciones precarias y vulnerables. En este sentido, dice Gootenberg (2010) que se trata de una desigualdad duradera, no de un fenómeno coyuntural.

La desigualdad también puede ser definida como un proceso multidimensional y relacional. Reygadas (2008) sostiene que se trata de una distribución asimétrica de las ventajas y desventajas de una sociedad. Para él, la desigualdad no se refiere únicamente a cuestiones económicas, sino que atañe a todos los aspectos de la vida: sociales, culturales, simbólicos y territoriales, entre otros. Entonces, las desigualdades más sustanciales son las que tienen que ver con las diferencias en las libertades para alcanzar los propósitos que cada uno tenga; por eso, pone en el centro el tema de las capacidades (Sen, 1999). Desde la perspectiva económica del autor bengalí, la igualdad política es fundamental para poder alcanzar la igualdad en otros terrenos (Sen, 1999, 2004). En relación con eso, y si bien con otras consecuencias por la importancia que el autor da en su obra al bienestar (y por cómo lo define), el lugar que Sen otorga a los procesos políticos, como condición previa o fundacional para alcanzar la equidad

6 Todos los nombres de los entrevistados fueron modificados para mantener el anonimato de los informantes. Solo son verdaderos la edad y el género. 
en otras dimensiones, se acerca a una de las tendencias principales de abordaje de la comunicación para el desarrollo (Beltrán, 2009) en América Latina (Chachagua, 2019).

Las diferentes investigaciones acerca de la desigualdad ponen en discusión qué "horizonte de igualdad" se presupone o se pretende. En ese marco, Kessler, en su libro Controversias sobre la desigualdad (2014), cita a Dubet (2011) para caracterizar los horizontes diferenciales entre igualdad de posiciones e igualdad de oportunidades. La igualdad de posiciones se refiere a que las personas o grupos de personas ocupen lugares en la estructura social cuyos beneficios sean más o menos similares. En otras palabras, desde esta perspectiva, se busca aproximar/acercar las distintas posiciones en la estructura social para que todas queden más cercanas entre unas y otras. En cambio, la igualdad de oportunidades está más relacionada con la idea de meritocracia, y se basa en asegurar que todos puedan competir en igualdad de condiciones por los lugares más deseables de la estructura social. Por tanto, la meta sería la competencia perfecta, sin que ningún vicio de origen y ningún rasgo propio conlleven algún tipo de discriminación. De esta manera, las inequidades no aparecen como un problema, ya que son parte de la estructura que se plantea como horizonte, en la que todas las posiciones están abiertas a todos. Kessler (2014) coincide en definitiva con Dubet en que es más justa una sociedad que tienda a la igualdad de posiciones (también denominada igualdad de lugares), ya que hay situaciones históricas que fundamentan que este "modelo" ha beneficiado a los más débiles y ha forjado sociedades más justas. No es un aspecto menor que la ejemplificación se realice a partir de experiencias estatales nacionales, por la importancia que las competencias del Estado y sus políticas tienen para abordar el problema en las sociedades contemporáneas desde la primera mitad del siglo xx (Chachagua, 2019). Por el contrario, el modelo de la igualdad de oportunidades puede implicar la justificación de ciertas desigualdades en la medida que hayan sido el resultado de una "competencia justa" (Kessler, 2014, p. 48).

Así, la desigualdad puede ser definida como un proceso multidimensional, y con esto Reygadas (2008) se refiere a que las diferencias económicas entre las personas se encuentran estrechamente vinculadas a la clase social, el género, la etnia y otras formas de estratificación social. Y, además, porque la desigualdad es "una cuestión de poder" (Reygadas, 2008, p. 34). Esto nos permite pensar que esta idea está relacionada con las asimetrías en la distribución de recursos y capacidades, y con las relaciones de poder que se establecen sobre la base de esas asimetrías.

En su investigación Kessler (2014) menciona el trabajo de Francisco Gatto, quien estudia la acumulación de desventajas familiares y territoriales en localidades y departamentos de once provincias del noreste (NEA) y noroeste (NOA) de Argentina. Este autor estima que en su país hay 900000 hogares con 4 millones de personas en una situación de pobreza crónica, lo que se fundamenta en la mala o nula inserción laboral, pero sobre 
todo por las carencias de infraestructura básica, tales como los servicios de luz, agua, salud, y la ausencia o insuficiencia de fuentes de trabajo locales (Gatto, 2007). Teniendo en cuenta estos y otros datos aportados en su obra sobre la desigualdad en Argentina entre los años 2003-2013, Kessler (2014) señala que "las mayores brechas en el período estudiado se advierten en las provincias del NOA" (p. 209).

Estas brechas son evidentes cuando se analizan algunos datos del último censo nacional (INDEC, 2010). Según estas estadísticas, Salta tiene 1214441 habitantes, de los cuales 1057951 (87,11\%) pertenecen a la población urbana y 156490 (12,89 \%) a la rural. Esta última se divide en agrupada, con 59104 personas (37,77 \%), y dispersa, con 97386 personas $(62,23 \%)^{7}$. En ese conjunto de población, primordialmente urbana, un dato que no es menor para la investigación que aquí se presenta es que el $16,9 \%$ de los hogares urbanos y el $36,6 \%$ de los hogares rurales tienen necesidades básicas insatisfechas (NBI). Por tanto, a partir de estas estadísticas se puede afirmar que la población rural en Salta con NBI es porcentualmente la más alta del país ${ }^{8}$ (Chachagua, 2019).

La provincia de Salta se ubica a 1187 metros sobre el nivel del mar y pertenece al noroeste argentino. Se estructura administrativamente en 23 departamentos, subdivididos en 58 municipios y 2 delegaciones municipales. La capital es la ciudad de Salta y es uno de los destinos turísticos más requeridos en el país y en el mundo. La denominan "Salta, la linda"; incluso el eslogan principal del turismo oficial generado por el gobierno provincial es "Salta, tan linda que enamora". Su nombre deriva de la palabra aimara sagta, que significa "la muy hermosa". Sin embargo, Salta no es tan hermosa si se observa la realidad en la que viven los ciudadanos. Actualmente la provincia presenta un panorama contextual muy complejo, atravesado por los agronegocios, la explotación de los campos, la inserción de empresas multinacionales que contaminan, las inundaciones, los altos índices de pobreza, desocupación, pobreza infantil, desnutrición, denuncias por violencia

7 La población urbana se refiere a la población que reside en áreas de 2000 o más habitantes. La población rural puede ser población agrupada (aquella que habita en localidades con menos de 2000 habitantes) o dispersa (que reside en campo abierto, sin constituir centros poblados).

8 El indicador de necesidades básicas insatisfechas (NBI) se construye a partir de la medición de aquellos hogares que presentan al menos una de las siguientes condiciones de privación:

(1) Vivienda: es el tipo de vivienda que habitan los hogares que moran en habitaciones de inquilinato, hotel o pensión, viviendas no destinadas a fines habitacionales, viviendas precarias y otro tipo de vivienda. Se excluye a las viviendas tipo casa, departamento y rancho.

(2) Condiciones sanitarias: incluye a los hogares que no poseen retrete.

(3) Hacinamiento: es la relación entre la cantidad total de miembros del hogar y la cantidad de habitaciones de uso exclusivo del hogar. Operacionalmente se considera que existe hacinamiento crítico cuando en el hogar hay más de tres personas por cuarto.

(4) Asistencia escolar: hogares que tienen al menos un niño en edad escolar (6 a 12 años) que no asiste a la escuela.

(5) Capacidad de subsistencia: incluye a los hogares que tienen cuatro o más personas por miembro ocupado y que tienen un jefe que no ha completado el tercer grado de escolaridad primaria (INDEC 2010). 
de género y femicidios, etcétera (Chachagua, 2019). "La muy hermosa" es noticia constantemente, pero no por lo "linda" (como venden los spots publicitarios y turísticos), sino por ser una de las provincias más desiguales del país por las diferentes problemáticas mencionadas y por las estadísticas oficiales que lo confirman.

\section{LO DIGITAL EN UN CONTEXTO SALTEÑO DESIGUAL}

Uno de los aspectos de la desigualdad como proceso multidimensional y relacional son las grandes disparidades que existen en el ámbito digital, y este proceso no puede explicarse únicamente por la división entre quienes tienen acceso a las nuevas tecnologías y quienes no lo tienen. Las desigualdades digitales se ponen de manifiesto en una serie de factores que van desde el acceso a un dispositivo digital, las competencias o las habilidades digitales, hasta los usos y las apropiaciones tanto de los dispositivos digitales como de internet. Así pues, las escalas que confluyen en la estructura de las desigualdades digitales son diversas y múltiples. Por tanto, la escala global de operación de las TIC es una complicación añadida a cualquier situación de desigualdad digital: si bien la distribución de todo recurso es afectada y determinada por la desigualdad estructural latinoamericana, las actividades informacionales y comunicacionales, en particular, se hallan reguladas no solo por condiciones relativamente endógenas propias de la historia y las condiciones presentes de los países de la región, sino también por un tipo de interconexión que es global y que supone la presencia de instituciones y de actores industriales y financieros de un sistema igualmente globalizado (Becerra, 2015).

Crovi Druetta (2004) plantea que el acceso desigual al mundo digital es un problema compartido en los países periféricos, y señala que existen al menos cinco dimensiones desde las cuales observar el abismo o brecha digital: "tecnología/infraestructura y su grado de actualización; habilidades o saberes digitales de los individuos; información (que incluye sobreinformación y desinformación); económica; participación democrática y global" (p. 17).

En cambio, la perspectiva de Alva de la Selva (2012) circunscribe el concepto de discurso promocional, el cual presenta a la sociedad de la información como aquella que a través de la tecnología llegará al progreso y la democracia, bajo el supuesto de que toda la humanidad podrá ser partícipe de ella (p. 19). Si lo pensamos en términos actuales, es cierto que la expresión "todos" es utópica, ya que la mera existencia de conectividad o acceso a computadoras u otro dispositivo digital no implica la disminución de la brecha digital. Además, en muchos países latinoamericanos basta con alejarse unos pocos kilómetros de las capitales para perder todo tipo de conexión. Un ejemplo de ello es la Escuela Rural Mediada por TIC, que vamos a abordar más adelante en este artículo. 
En términos de acceso, la ejecución del Programa Conectar Igualdad ${ }^{9}$ en Argentina (específicamente entre el 2010 y el 2015) permitió que muchas familias y jóvenes accedan a una computadora portátil para poder estudiar; sin embargo, hubo diferentes usos y apropiaciones que excedieron "lo educativo", que también forman parte del contexto y la identidad de los destinatarios de esta política pública. A todo esto, Van Dijk (2005) señala que "la distribución de recursos tecnológicos que fundamenta el concepto de brecha digital crea diversas formas de acceso desigual, a través de los mecanismos de exclusión social, explotación y control" (p. 18). Este autor sostiene que la brecha digital se profundiza en la medida en que deja de ensancharse; es decir que, cuando una sociedad supera el acceso material a una gama (dispar) de bienes y servicios infocomunicacionales, las diferencias en su uso, las distintas modalidades de acceso a la segmentada oferta de servicios, así como la adquisición y construcción diferencial de capacidades y habilidades, se ahondan. Van Dijk (2005) afirma:

La brecha digital está lejos de ser superada. En la mayor parte del mundo, está aún ampliándose. La brecha entre países desarrollados y en desarrollo es extremadamente amplia, y está creciendo. Incluso en las sociedades más desarrolladas tecnológicamente, donde la división en el acceso físico detuvo su crecimiento, cerca de un cuarto o incluso un tercio de la población no tiene acceso a computadoras e internet. (p. 2)

Para este autor, hay que saber diferenciar entre los distintos tipos de acceso que conducen a una apropiación compleja de las nuevas tecnologías infocomunicacionales: el acceso motivacional, el acceso físico o material, las habilidades y, por último, el uso. El acceso material o físico se refiere a una terminal de recepción, uso o consumo (por ejemplo, computadora con acceso a redes, teléfono móvil con paquete de datos necesario para conectarse a internet), como una condición ineludible del acceso, así como lo es la motivación/interés para utilizar esta tecnología. Asimismo, la combinación entre factores económicos y materiales, y las diferentes modalidades de acceso y usos sociales de internet determinan brechas muy nítidas en los países latinoamericanos (Becerra, 2015).

9 En el 2010, en Argentina, el Poder Ejecutivo Nacional anunció que asumiría la responsabilidad de garantizar la inclusión digital, para lo cual lanzó el Programa Conectar Igualdad (Decreto 459/10), que es una política pública de inclusión digital basada en la modalidad "uno a uno" (una computadora, un niño) para la incorporación de las TIC en la educación. Este programa se destacó en la región por su carácter masivo en cuanto a la entrega de netbooks en todo el país. Según su fundamentación, su principal objetivo fue revalorizar y reposicionar a la escuela pública a través de una estrategia dirigida a reducir la brecha social, educativa y digital, mejorar los procesos de aprendizaje, actualizar las formas de enseñanza y fortalecer el rol docente. Hasta el 2015 se entregaron 5315000 computadoras en todo el país. Después, desde el 2016 hasta la disolución del programa en el 2018, no hay números oficiales que den cuenta de la entrega de equipamientos. 
El diseño y creación de una política como Conectar Igualdad, en su momento, advirtió que entendía a la escuela como un medio excepcional para democratizar el acceso al conocimiento (en un contexto de auge de las TIC). De allí que esa situación le generara al Estado la responsabilidad (y la obligación) de preparar al sistema educativo para que capacite a los estudiantes y docentes en la utilización comprensiva y crítica de las nuevas tecnologías, así como en la producción de contenidos digitales educativos. De esa forma, esta política no solo se presentaba como la posibilitadora del acceso y uso instrumental de las TIC, sino también de la enseñanza y aprendizaje de competencias digitales, y la apropiación de estos nuevos dispositivos digitales.

\section{HABLEMOS DE JUVENTUDES}

"Los jóvenes", "la juventud”, "las juventudes" son diferentes formas de nombrar a una categoría que históricamente fue considerada desde la edad como base de las clasificaciones sociales. Para Reguillo (2000), la "edad" es una dimensión que no puede entenderse de manera homogénea y estática; esto se debe a que asume valencias distintas entre las diferentes sociedades y también en el interior de cada una de ellas, ya que se establecen diferencias en función de los lugares sociales que los jóvenes ocupan. Por tanto, la noción de juventud actualmente ya no puede ser comprendida solo desde una dimensión etaria, sino que existen múltiples estudios que proponen un amplio abanico de perspectivas de abordaje. Así, Vommaro (2014a, 2014b) plantea que la juventud es una producción sociohistórica y cultural, situada y relacional. Por su parte, Margulis y Urresti (1996) afirman que no existe una única juventud. El concepto de juventud está cargado con diferentes evocaciones y significados, y puede conducir a laberintos del sentido si no se tienen en cuenta la heterogeneidad social y las diferentes modalidades con que se presenta en la sociedad y en la cultura la condición de joven. En consecuencia, atrás queda la referencia de edad, ya que se suman las dimensiones de la diferenciación social y la cultura. En efecto, Margulis y Urresti (1996) plantean que la juventud depende de una moratoria, es decir, un espacio de posibilidades abierto a ciertos sectores sociales y limitado a determinados períodos históricos. La noción de moratoria social da cuenta de que existen grupos de jóvenes que posponen la edad de matrimonio y de procreación (durante un tiempo cada vez más prolongado); algunos eligen estudiar o avanzar en su formación académica (posgrados, intercambios estudiantiles, pasantías laborales) apostando a desempañarse en ámbitos laborales exitosos, o simplemente deciden viajar, conocer el mundo y vivir experiencias nuevas. Por eso este concepto adhiere implícitamente a ciertos "límites" vinculados con la condición de juventud.

Esta etapa transcurriría entre el final de los cambios corporales que acaecen en la adolescencia y la plena integración a la vida social que ocurre cuando la persona forma un hogar, se casa, trabaja, tiene hijos. 0 sea, juventud sería el lapso que media entre la madurez física y la madurez social. (Chaves, 2009, p. 6) 
Esta condición de juventud varía de acuerdo con los diferentes contextos sociales, culturales y geográficos. La juventud, como el período en el que se posterga la asunción plena de responsabilidades económicas y familiares, se evidencia como una característica reservada para los sectores sociales con mejor posición económica. Pero ¿qué sucede con los jóvenes que no tienen esa posibilidad?

Margulis y Urresti (1996) critican la noción de moratoria social y la caracterizan como un etnocentrismo de clase, es decir, como una actitud que presupone su superioridad sobre los demás y hace de la cultura propia el criterio exclusivo para interpretar y valorar los comportamientos de una sociedad. En efecto, los autores proponen la idea de "moratoria vital", que refiere a una dimensión cronológica de la juventud; sería un aspecto objetivante de su definición que consiste en poseer mayor capital energético y sentirse (y estar) más alejado de la muerte que otras generaciones; a esto también se llama "crédito temporal o vital", lo que corresponde a más tiempo por delante para vivir.

\section{¿Y LAS JUVENTUDES RURALES?}

Las investigaciones existentes sobre juventudes en América Latina plantean características importantes para distinguir y diferenciar al joven rural (González Cangas, 2003). Este autor hace principal énfasis en determinantes económicos y sociales que harían al "supuesto joven" rural asumir funciones adultas rápidamente, debido a sus regímenes de matrimonios, maternidades y paternidades más tempranas, ausencia de períodos formativos escolares y una inserción laboral efectiva. Por esto, el tiempo de moratoria no existiría o se disminuiría considerablemente, sin alcanzar a formar un cuerpo social con identidad y convirtiendo a los sujetos en "campesinos de menos edad u obreros de menos edad” (González Cangas, 2003; Vio Grossi, 1986; Méndez, 1986).

La constitución de las juventudes rurales adquiere significados distintos con relación a las juventudes contemporáneas (que tienden a generalizarse para el conjunto). Muchos de ellos viven un período de moratoria específico y diferente en cuanto a moratoria, socialización, dinámica generacional, etcétera, que no siempre se da con características formativas en la escuela, sino que se vincula directamente con la incorporación temprana a las labores productivas, en el hogar o en el campo; estos sentidos pueden generar eventualmente cierta "identidad juvenil rural" (Díaz y Durán, 1986). Pero no todos los jóvenes se encuentran en la misma situación en los lugares donde no hay opciones para continuar estudiando; "salir a trabajar" no solo de debe a ser padre/madre, sino a que no hay otras opciones una vez concluida la educación primaria (Chachagua, 2019). En este contexto, la ERMT apunta a transformar esas prácticas naturalizadas, porque ahora los jóvenes de estos parajes rurales, como sujetos de derechos, tienen la posibilidad de continuar sus estudios secundarios (y la posibilidad de elegir, lo cual no es menor) para completar su ciclo de formación escolar. 


\section{RESULTADOS}

Si analizamos las categorías anteriores con los jóvenes que asisten a la ERMT, podemos decir que en la ruralidad salteña existe la heterogeneidad que plantean los autores. Una de las dimensiones que se abordaron durante el trabajo de campo (por encuestas y entrevistas) fueron las proyecciones a futuro o deseos de los estudiantes. Las respuestas dan cuenta de la situación compleja en que se encuentran por las condiciones geográficas, sociales y económicas, pero sobre todo aparece la condición de género marcando una desigualdad.

Algunos de los jóvenes entrevistados sobre sus proyecciones a futuro comentaron que ya trabajan (informalmente) o están buscando trabajo. Varios de ellos dijeron no estar conformes con trabajar, pero que es necesario para ayudar a sus familias. La idea de "conformidad" se presenta relacionada con la idea de "elección", pero con factores que la subordinan, como la situación económica familiar. La tabla 1 y la figura 2 muestran los resultados obtenidos a partir de una de las encuestas realizadas en la ERMT acerca de las proyecciones a futuro de los jóvenes.

Tabla 1

Resultados de la encuesta a jóvenes sobre proyección a futuro

\begin{tabular}{lc}
\hline Respuestas & Porcentaje \\
\hline Trabajar en el campo & $33 \%$ \\
Mudarse a otra ciudad & $11 \%$ \\
Ingresar a la Gendarmería & $22 \%$ \\
Estudiar para maestra jardinera & $6 \%$ \\
Estudiar para profesor(a) de inglés & $6 \%$ \\
Estudiar enfermería & $11 \%$ \\
Armar una familia/casarse & $11 \%$ \\
\hline
\end{tabular}

Elaboración propia 


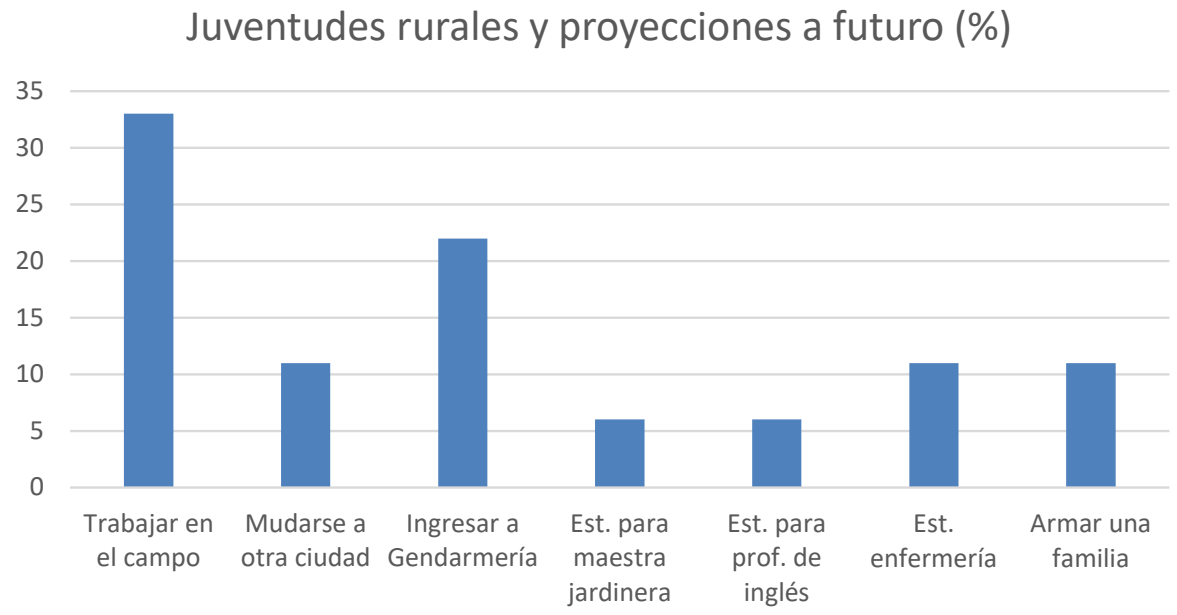

Figura 2. Resultados de la encuesta a jóvenes sobre proyección a futuro

Elaboración propia a partir de las encuestas realizadas durante el trabajo de campo en el año 2017 (Chachagua, 2019)

Como se observa, la respuesta más elegida fue la de trabajar en el campo (33\%); en segundo lugar, se encuentra la opción de ingresar a la Gendarmería (22 \%); luego, con un $11 \%$ para cada respuesta: mudarse a otra ciudad o provincia, armar una familia o estudiar enfermería. Finalmente, también emerge la respuesta de estudiar para maestra jardinera o profesor(a) de inglés. El deseo de salir del hogar puede estar relacionado con la idea de independencia económica, pero también de cambiar sus propias historias: "para mí sería importante mudarme a la ciudad y buscar trabajo allí" (Eugenia, 13 años, estudiante).

Pero también lo dice el "deseo" de sumarse a alguna fuerza, por ejemplo, la Gendarmería. Ese anhelo por independizarse se enfrenta con la compleja situación económica en Argentina, donde el desempleo ha crecido fuertemente; en el tercer trimestre del 2019 las estadísticas indicaban un 9,7 \% de desempleo, según INDEC. De esta manera, la elección radica para algunos en la vocación, pero muchos otros lo hacen por la "estabilidad" económica que proponen estas instituciones. También es importante señalar que hay situaciones en que los jóvenes se encuentran "presionados" por las condiciones precarias que viven sus familias, por lo que necesitan un ingreso económico para colaborar con sus padres, madres, hermanos, hijos, etcétera. Para algunos, la Gendarmería es una "salida laboral rápida y segura", por lo que eligen esta opción para no fracasar. Aunque hay quienes también reconocen que no es nada fácil, porque la preparación en estas instituciones es muy exigente y "hay que bancársela". 
Creo que es la salida laboral más conveniente en esta época que no hay trabajo en ningún lado. (Manuel, 17 años, estudiante)

Dicen que se sufre mucho por la distancia y las pruebas que exigen, pero vale la pena para salir adelante. (Cecilia, 17 años, estudiante)

No menos importante es que otros estudiantes expresan como expectativa de futuro "casarse" o "tener un familia" para salir de la casa.

El papá de mi nena se fue a la ciudad a buscar trabajo, porque aquí no conseguía; por ahora yo vivo con mis padres y hermanos, hasta que mi novio nos pueda llevar lejos. (Leila, 20 años, estudiante)

Hay jóvenes que pueden elegir entre estudiar o trabajar, pero otros no tienen opción, ya que deben adaptarse a las condiciones contextuales en las que viven: muchos jóvenes rurales trabajan en la casa (sobre todo las mujeres) y en la cosecha o cuidado de animales (sobre todo los varones). En ese escenario desigual, se presenta una diferencia mayor que es la de género.

La desigualdad de género se profundiza aún más cuando la joven mujer es madre y vive en un contexto rural, donde la única opción que ve como posible es "salir de la mano de su pareja a un lugar mejor". Una de las jóvenes estudiantes de la ERMT, Leila (20 años), quedó embarazada a los 17 años cuando apenas ingresaba a la escuela; al principio abandonó por decisión propia (según su relato) y luego decidió regresar cuando nació su hija ${ }^{10}$. Leila cuenta que tiene una prima de su misma edad y que es mamá, quien decidió quedarse en el paraje rural debido a que el padre de su hijo tiene trabajo en el campo. En cambio, "nosotros (por Leila y su novio) queremos otra vida para nuestra hija". A la escuela rural asisten jóvenes embarazadas y madres, ¿qué pasa con ellas y su maternidad? ¿Cómo les cambia la vida en términos sociales, económicos y educativos?

La Ley Nacional N.² 25.584, sancionada en el 2002, establece la prohibición en establecimientos de educación pública de acciones que impidan el inicio o la continuidad del ciclo escolar a estudiantes embarazadas. Además, en el 2003, se promulgó la Ley Nacional $\mathrm{N}^{\circ}{ }^{\circ} 25.808$, que incluye a los colegios privados dentro de esta normativa. Así pues, las mujeres embarazadas (sin importar la edad) pueden continuar estudiando sin que la institución tome alguna medida en su contra. Sin embargo, la realidad que viven las jóvenes es mucho más compleja. Obviamente es importante que la institución (y el Estado) las respalde, pero además las mujeres viven diferentes transformaciones tanto

10 El último informe de natalidad y mortalidad publicado por el Ministerio de Salud de la Nación (2017) muestra que en Salta el 22,3\% de los nacimientos corresponden a madres menores de 20 años, muy por encima del promedio nacional, el cuarto más alto de Argentina. Amnistía Internacional reveló que en el 2017 se registró en el país un total de 72791 embarazos adolescentes no deseados, lo cual representa una tasa de 41,9 por cada 1000 mujeres de entre 15 y 19 años. 
psicológicas como físicas provocadas por el embarazo, el parto y el posparto: algunas se enferman y tienen que hacer reposo, a otras les tocó abandonar la escuela para salir a trabajar, entre otras múltiples situaciones.

En una de las visitas a la escuela rural, en la que se abordó justamente la diferencia de géneros, se entrevistó a Amalia, una de las ordenanzas de la ERMT, quien se caracteriza por ser un poco tímida y con dificultades para expresarse; sin embargo, ella comenta que cada estudiante "es un mundo".

Trabajo hace tres años aquí, mi puesto no es muy lejos, así que voy y vengo todos los días [...] de chica estudié en esta escuela, pero no pude hacer la secundaria porque en ese tiempo no había. Mi nene (Matías, de 4 meses) nació aquí, y lo traigo todos los días en el coche, ya está acostumbrado, los chicos me ayudan a cuidarlo en los recreos. (Amalia, 25 años, ordenanza, entrevista realizada en junio del 2017)

Este relato muestra que Amalia forma parte de una generación de jóvenes que no pudieron elegir (más allá de sus condiciones sociales y económicas en ese momento) si querían estudiar/continuar estudiando o no, ya que en ese momento no existía la ERMT y la Ley Nacional de Educación (que establece como obligatoria la educación secundaria para todos los jóvenes del país) tampoco se cumplía; por lo tanto, no tenían posibilidades de estudiar el nivel secundario en esos parajes rurales.

Por lo anteriormente expuesto, se puede decir que las mujeres están sometidas a una exclusión social más severa, no solo en cuanto al acceso y la apropiación digital para la educación, sino en lo que concierne a las condiciones de vida específicamente. Este trabajo, en términos de desigualdad, intenta aportar a la reflexión acerca de la diferencia de género en ámbitos rurales, donde no alcanza con el acceso a las tecnologías digitales para estudiar; se necesita de más acompañamiento para que las mujeres puedan llegar y mantenerse en la escuela, terminar el secundario y pensar en otros futuros posibles.

Tanto Amalia (25 años, ordenanza) como Leila (20 años, estudiante) son mamás de niños pequeños. La pequeña hija de Leila va a clases con su mamá todos los días, su cochecito siempre está en el aula y nunca faltan sus "tíos y tías del corazón" que ayudan en su cuidado. "Se porta bien y nos encanta tenerla aquí", dice Larisa (17 años, estudiante, entrevista realizada en junio del 2017), compañera de mesa de la joven madre. Hay otra estudiante que también tiene una hija; la niña va a primer grado, en un aula que está ubicada frente al salón que corresponde al secundario, así que también se ven frecuentemente. La maestra a veces tiene que salir a buscarla porque se va a ver a su mamá, se queda jugando y no regresa.

Estas jóvenes son madres y van a la escuela con sus hijos, es decir que toman distancia del modelo hegemónico de la mujer "casada", "madura” y "preparada" para esta función. Leila fundamenta su esfuerzo para continuar en la escuela solo para ofrecerle 
un mejor futuro a su hija. El mandato cultural dominante de "ser madre" recae sobre toda mujer sin importar la clase (Mancini, 2004), aunque su significado adquiere diferentes características según el sector social y las diferentes culturas. Marcus (2006) plantea que la situación de precariedad material en la que se vive en ciertos espacios de socialización (por ejemplo, el rural), así como las pocas posibilidades de realización laboral y crecimiento profesional, terminan imponiéndose y estableciendo que la maternidad se presente como una vía de afirmación y realización personal. A estas condiciones materiales de existencia se suman mandatos y pautas culturales que refuerzan el valor positivo de la maternidad. Muchas veces los embarazos no son planificados ni buscados por estas jóvenes mujeres, y junto al sentimiento de gratificación que supone ser madre se superpone otro: el de una aceptación a veces resignada como un destino inherente al ser mujer (Marcus, 2006). En estos sectores la maternidad temprana es culturalmente más aceptada, así como la cantidad de hijos por mujer suele ser bastante más elevada que en los sectores medios (Chachagua, 2019).

La desigualdad de género se presenta en muchas de las sedes de la escuela rural. Según el último relevamiento estadístico de Unicef (2019), hay 210 estudiantes en total en todas las sedes de la ERMT, la mayoría de la cuales son varones (57\%) y el $43 \%$ son mujeres.

Tabla 2

Cantidad de estudiantes de la ERMT dividida por género

\begin{tabular}{|l|l|}
$\mid$ Total de estudiantes en todas las sedes de la ERMT \\
\hline Varones & $57 \%$ \\
\hline Mujeres & $43 \%$
\end{tabular}

Elaboración propia a partir de los datos del Relevamiento Unicef (2019) 


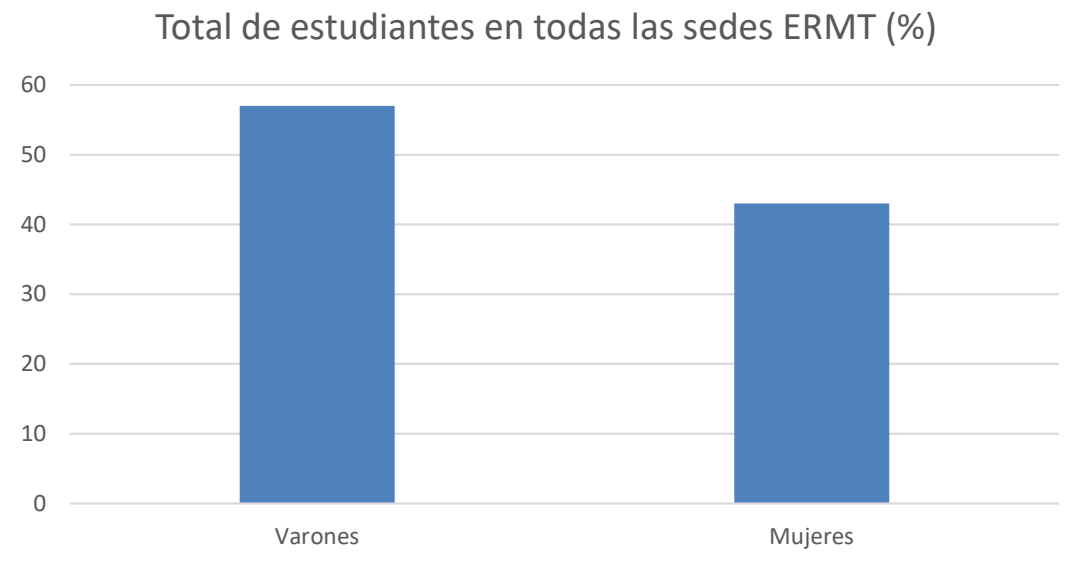

Figura 3. Cantidad de estudiantes de la ERMT dividida por género

Elaboración propia a partir de los datos del Relevamiento Unicef (2019)

Para ser más específicos, la tabla 4 y en la figura 3 expresan la cantidad de mujeres y varones por sede.

Tabla 3

Estudiantes por género, por sede

\begin{tabular}{lcc}
\hline \multicolumn{1}{c}{ Sedes } & \multicolumn{2}{c}{ Estudiantes } \\
& Varones & Mujeres \\
\hline La Argentina & 8 & 2 \\
Campo Durán & 7 & 7 \\
Esquina de Guardia & 5 & 5 \\
El Trementinal & 5 & 4 \\
Los Pozos & 6 & 6 \\
Media Luna & 13 & 8 \\
Madrejones & 6 & 3 \\
Pucará & 25 & 8 \\
Santa Teresa & 10 & 10 \\
El Cuarto & 11 & 14 \\
El Mirador & 5 & 9 \\
Ciervo Cansado & 6 & 7 \\
Pozo de la Piedra & 8 & 3 \\
Pelícano Quemado & 4 & 5 \\
\hline
\end{tabular}

Elaboración propia a partir de los datos del Relevamiento Unicef (2019) 


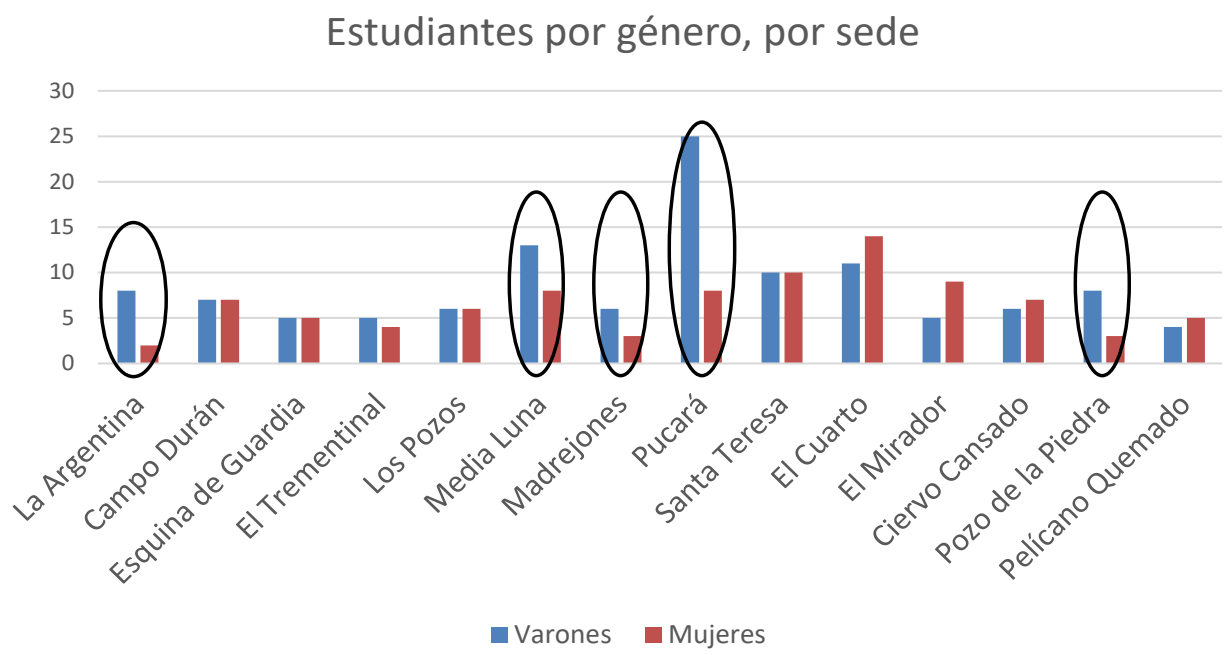

Figura 4. Cantidad de estudiantes de la ERMT dividida por género

Elaboración propia a partir de los datos del Relevamiento Unicef (2019)

Se puede observar que la cantidad de estudiantes por sede es heterogénea. En la mayoría de las sedes, el número de mujeres es menor que el de varones; se destaca el caso de La Argentina, a la que asisten solo dos mujeres, y el de Pucará, donde asiste menos de un tercio de mujeres con respecto a los varones. Además, así como Leila (estudiante), el Relevamiento Unicef (2019) arroja que 18 de los 210 estudiantes que son en total tienen hijos, y una (1) de ellas está embarazada. La mayoría de ellos solo tienen un (1) hijo. Una estudiante tiene tres (3) hijos. Otra estudiante tiene (4) hijos. Dos estudiantes tienen 5 hijos. Y 8 de los 18 estudiantes llevan a sus hijos a la escuela.

\section{CONCLUSIONES}

En este artículo nos posicionamos desde la perspectiva del derecho y la inclusión social (y digital), desde el anhelo de un modelo de desarrollo de bienestar, en donde el Estado sea el protagonista central, tanto para regular la economía como para respaldar y garantizar derechos para todos los ciudadanos.

Salta es una de las provincias argentinas con mayores niveles de desigualdad vigente; para ser más específicos, el $36,6 \%$ de los hogares rurales tienen necesidades básicas insatisfechas, cifra que es porcentualmente la más alta del país. Como señalan los autores citados, la desigualdad es un proceso multidimensional, relacional; se trata de un fenómeno duradero y no coyuntural. Además, no se refiere únicamente a 
cuestiones económicas, sino que atañen otros aspectos como los sociales, culturales, de género y digitales.

La Escuela Rural Mediada por TIC surge como respuesta a la demanda de la población de contar con educación secundaria en estas zonas. Si bien por medio del $\mathrm{PCl}$ y de Unicef, los jóvenes accedieron a sus computadoras personales y a internet para estudiar, comunicarse con sus docentes y descargar el material educativo, no es suficiente para lograr la igualdad. La brecha digital plantea diferentes dimensiones (Crovi Druetta, 2004) para tener en cuenta:

1. Se trata del acceso a la infraestructura y a los dispositivos tecnológicos. La ERMT cuenta con computadoras, routers, impresoras y pantallas, pero la conexión a internet no es buena. Los estudiantes indican que a veces pueden conectarse muy bien y otros días es imposible. A esto se le suma que, al no tener luz eléctrica, recargar las computadoras portátiles implica demoras y problemas a diario.

2. Está relacionado con las habilidades y saberes tecnológicos. La cuestión que se debate aquí, y que no queremos dejar de nombrar, es si como plantean algunos autores (como Prensky, 2001) los jóvenes son "nativos digitales" y los adultos, "inmigrantes digitales". Pero esta teoría es un tanto positivista, ya que no se consideran las dimensiones de clase y género, la desigualdad persistente en el mero acceso, etcétera. La realidad es mucho más compleja, cada estudiante y docente es distinto. Por ejemplo, ellos refieren ${ }^{11}$ que hay trabajos prácticos que pueden hacer sin problemas y otros que se les dificulta por el uso de algunos programas, como los de matemática o química, para los cuales necesitan la explicación y acompañamiento de los profesores o celadores (Chachagua, 2019).

3. La información es pública, pero no todos los ciudadanos tienen la misma posibilidad de acceso. Internet es una puerta central para acceder a información de cualquier índole, pero la falta de una buena conexión causa que el acceso esté en la cuerda floja. Sin embargo, la instalación de una antena de internet en la escuela no solo revolucionó a los jóvenes que quieren estudiar, sino también a los vecinos de la zona, quienes se acercan a la escuela para conectarse al wifi, ingresar a alguna red social, hacer un trámite en línea, etcétera. Esa posibilidad se hizo realidad por esta política de educación que trajo añadida la incorporación de internet en el paraje rural.

4. La dimensión económica pone de manifiesto el acceso desigual de las tecnologías. Por ejemplo, en cuanto al uso de celulares, son muy pocos los estudiantes

11 Información recogida de las encuestas realizadas a los estudiantes sobre usos educativos de la netbook (Chachagua, 2019). 
que poseen uno propio. En el momento que se hizo el trabajo de campo, en la sede Santa Teresa, solo dos estudiantes tenían un celular, pero no era propio, sino compartido con su familia ${ }^{12}$. Los vecinos del lugar siempre estuvieron conformes con la instalación de la antena de internet, ya que ahora sí podían tener un celular para darle un uso más dinámico y comunicarse por WhatsApp ${ }^{13}$ Esta dimensión también tiene que ver con la falta de los servicios básicos como el agua potable, la luz eléctrica, el gas natural, etcétera.

5. La última dimensión se refiere a la participación democrática y global. En este sentido, la ERMT está promoviendo poco a poco diferentes instancias de participación de los jóvenes en foros, centros de estudiantes en línea, entre otras actividades que les permiten comunicarse con otros jóvenes de las demás sedes para poner en común sus necesidades, reclamos y también sugerencias.

El análisis de las cinco dimensiones planteadas por Crovi Druetta (2004) evidencia que el acceso a las tecnologías es desigual y es un problema que supera a la ruralidad, porque tiene que ver con otros factores que implican a las decisiones políticas de los gobiernos y a las organizaciones que tratan esta temática. De esta manera, la desigualdad digital se suma a las desigualdades ya persistentes en el lugar, lo que profundiza aún más la problemática.

Por otra parte, como dijimos anteriormente, ya no se considera a los jóvenes solo por la edad, sino por el lugar que ellos ocupan en la sociedad (por eso la categoría es una producción sociohistórica, cultural, situada y relacional). Y esta condición varía también en la ruralidad. Hay jóvenes que son padres o madres, otros que trabajan en el campo o en las tareas domésticas de los puestos, otros que quieren estudiar y otros que lo hacen por obligación; hay quienes quieren seguir estudiando, ser profesionales, y otros que quieren salir de los parajes para buscar "una vida mejor". En esas múltiples y diversas posibilidades y características se halla la heterogeneidad a la que nos referimos. Esta investigación indagó sobre un conjunto de estudiantes de la misma escuela y del mismo lugar, donde comparten características, pero también se diferencian entre sí. Los jóvenes conviven e interactúan en distintos ámbitos (familia, escuela, etcétera), allí construyen y (re)construyen las identidades, y se definen las formas de ser joven.

Las modalidades sociales del ser joven, dice Margulis (2008), dependen de la edad, el crédito vital, la clase social, el marco institucional y el género. Entonces, no se manifiestan de la misma manera si el joven forma parte de una clase social más vulnerada,

12 Información recogida de las encuestas realizadas a los estudiantes sobre acceso a teléfonos móviles (Chachagua, 2019).

13 En esa zona no hay señal telefónica; por tanto, no se pueden hacer llamadas ni mandar mensajes de texto. 
porque los recursos que brinda la moratoria social no están distribuidos de manera igualitaria entre los habitantes de los diversos sectores sociales. "Esto significa que la ecuación entre moratoria y necesidad hace probablemente más corto el período juvenil en sectores populares y más largo en las clases medias y altas" (Margulis, 2008, p. 10). Esta situación se suma a las condiciones de género: "Hay más probabilidades de ser joven siendo hombre que siendo mujer, ya que los hijos implican urgencias distintas en la inversión del crédito social disponible" (Margulis, 2008, p. 10).

Por lo anterior, en este artículo se decide denominar por el plural al concepto de juventudes. Esta elección debe ser interpretada, no como un neologismo banal, sino "como una lucha política" de afirmación de la heterogeneidad en oposición al discurso homogeneizador (y dominante) que prevalece en las investigaciones previas sobre juventud en Argentina, que además continúa guiando muchas de las políticas de intervención en el sector y que articula uno de los significados más reproducidos en torno a los jóvenes, invisibilizando la complejidad de sus vidas (Chaves, 2009, p. 5). Sumado a esto, y no menos importante, las juventudes son sujetos de derecho. Por lo tanto, las políticas públicas, como el $\mathrm{PCl}$ o la educación mediada por TIC, no deben ser consideradas como un "beneficio", sino precisamente como un derecho al cual los jóvenes acceden en su condición de ciudadanos y ciudadanas.

Para finalizar este apartado, y retomando la discusión teórica inicial acerca de los horizontes de igualdad que anhelamos, podemos decir que la ERMT es una política que apunta a que los jóvenes de los distintos parajes rurales puedan acceder a la educación secundaria en condiciones más o menos similares (como formas de enseñanza y aprendizaje, de evaluación y seguimiento, acceso a una alimentación saludable y completa durante el día, los mismos dispositivos tecnológicos para todos, entre otros) y se asemeja a la propuesta de igualdad de posiciones que plantea Dubet (2011). Desde esta perspectiva, se intenta acercar las distintas posiciones en la estructura social, para que todas queden más próximas, las unas a las otras. Así es posible que, más allá de las distancias geográficas y las desigualdades persistentes, los jóvenes puedan estudiar mediante la tecnología y finalizar su ciclo escolar.

\section{CONTRIBUCIONES: LAS UTOPÍAS SIRVEN PARA CAMINAR}

A lo largo del trabajo se han analizado diferentes ejes que permiten pensar y soñar una sociedad más igualitaria, en donde se diseñen y apliquen políticas públicas emancipadoras, puesto que el Estado es central como garante de los derechos, y hay que luchar por ello.

Como mencionamos en el apartado anterior, entendemos a la ERMT como una política que aspira a la igualdad de posiciones. Creemos que es pertinente mejorarla para que 
sea una política pública garantizada por el Estado, en conjunto con otros organismos como Unicef, y que no apunte a la meritocracia ni a la competitividad entre estudiantes por ser "el mejor".

En cuanto a lo tecnológico, es importante reforzar la inversión realizada para la conexión a internet, para que tenga un espectro de banda ancha más potente y estable no solo para los usos educativos, sino para los pobladores en general, con el objetivo de mejorar los procesos de comunicación.

En cuanto a la desigualdad de género, se observa que hay estudiantes que por ser madres o simplemente por ser mujeres, o por mandatos tradicionales y patriarcales tienen que hacer tareas domésticas, cuidados de niños y ancianos, etcétera. Esto les impide disponer de un tiempo máximo para estudiar y progresar académicamente. Incluso los datos del Relevamiento Unicef (2019) dan cuenta de la desproporción de mujeres estudiantes en relación con varones estudiantes, en muchas de las sedes de las ERMT. Algunas mujeres ni siquiera llegan a la escuela, ya sea por desconocimiento o simplemente por no sentirse preparadas o convocadas a completar sus estudios. Esta dimensión es central para pensar la inclusión social y educativa, y para lograr una sociedad más justa. La cuestión del cuidado debe ser una cuestión de Estado (y no de "amor"), es decir que se pueda garantizar espacios de resguardos para los hijos cuando sus madres estudian, por ejemplo. Otra propuesta pueden ser las licencias estudiantiles para las mamás y los papás; así el nacimiento de un hijo(a) no sería un "obstáculo" o una razón para dejar la escuela, sino que podrían tomarse licencias por tiempos determinados y luego reincorporarse para no perder la continuidad de los estudios. La modalidad de mediación tecnológica permite esta posibilidad; por lo tanto, estas propuestas no son tan ajenas a la realidad, son posibilidades concretas que deben ser impulsadas desde los Estados y organismos intervinientes. Es una demanda que surge de los estudiantes, quienes en sus relatos cuentan sus necesidades, sus problemas para asistir y continuar en la escuela, etcétera. Al realizar el cruce entre la información recogida en el trabajo de campo y el cuerpo teórico propuesto, llegamos a estas conclusiones que nos permiten reflexionar y proponer ideas que tienen como punto de partida los sueños y los deseos colectivos (Merello, 1973).

Pensar y querer un mundo mejor no es solo una utopía, sino que puede ser una construcción colectiva gestada desde los propios actores, pero también en relación con cientos de personas que viven en la misma situación en América Latina. Socializar la experiencia de la ERMT y el PCI como política de inclusión permite, además, expandir las posibilidades de acción del Estado para garantizar la educación, y luego desde allí poder luchar juntos por más garantías y derechos. Seguramente habrá gobiernos con perspectivas neoliberales que proponen otros modelos de desarrollo, con los cuales entran en tensiones estas posibilidades; sin embargo, la organización y lo colectivo es lo que finalmente permitirá no abandonar nunca los sueños (y las utopías). 


\section{REFERENCIAS}

Alva de la Selva, A. R. (2012). Brecha e inclusión digital en México: hacia una propuesta de políticas públicas. Universidad Nacional Autónoma de México.

Becerra, M. (2015). De la concentración a la convergencia. Políticas de medios en Argentina y América Latina. Buenos Aires: Paidós.

Beltrán, L. R. (2009). La comunicación para el desarrollo en Latinoamérica: un recuento de medio siglo. En D. Loreti, G. Mastrini y M. Baranchuk (Comps.), Participación y democracia en la sociedad de la información (pp. 149-188). Buenos Aires: Prometeo.

Chachagua, M. (2019). Políticas de diseminación tecnológica, escuelas y jóvenes: sentidos de las TIC en el campo y la ciudad (Salta, Argentina, 2015-2017) (tesis doctoral). Universidad Nacional de La Plata, Argentina. Recuperado de http://sedici.unlp. edu.ar/handle/10915/73552

Chaves, M. (2009). Estudios sobre juventudes en Argentina I. Universidad Nacional de La Plata; Red de Investigadora/es en Juventudes Argentinas.

Crovi Druetta, D. (2004). Sociedad de la información y el conocimiento. Algunos deslindes imprescindibles. En D. Crovi Druetta (Coord.), Sociedad de la información y el conocimiento. Entre lo falaz y lo posible (pp. 17-56). Buenos Aires: La Crujía Ediciones.

Díaz, C., y Durán, E. (1986). Los jóvenes del campo chileno, una identidad fragmentada. Santiago de Chile: GIA.

Dubet, F. (2011). Repensar la justicia social: contra el mito de la igualdad de oportunidades. Buenos Aires: Siglo Veitiuno Editores.

Gatto, F. (2007). Crecimiento económico y desigualdades territoriales. Algunos límites estructurales para lograr una mayor equidad. En B. Kosacoff (Ed.), Crisis, recuperación y nuevos dilemas. La economía argentina, 2002-2007 (pp. 308-357). Buenos Aires: Comisión Económica para América Latina y el Caribe.

Gentili, P. (2009). Marchas y contramarchas. El derecho a la educación y las dinámicas de exclusión incluyente en América Latina. Revista Iberoamericana de Educación, 49, 19-57.

González Cangas, Y. (2003). Juventud rural, trayectorias teóricas y dilemas identitarios. Nueva Antropología, 19(63), 153-175.

Gootenberg, P., y Reygadas, L. (Eds.). (2010). Indelible inequalities in Latin America. Insights from history, politics, and culture. Durham: Universidad de Duke Press. 
Instituto Nacional de Estadística y Censos (INDEC). (2010). Datos estadísticos del Censo.

Kessler, G. (2014). Controversias sobre la desigualdad. Buenos Aires: Fondo de Cultura Económica.

Ley $\mathrm{N}^{\circ}$ 25.584, Prohíbase en los establecimientos de educación pública toda acción institucional que impida el inicio o continuidad del ciclo escolar a alumnas embarazadas. (2 de mayo del 2002). Recuperado del sitio de internet de InfoLEG: http://servicios.infoleg.gob.ar/infoleglnternet/anexos/70000-74999/74073/ norma.htm

Ley N..$^{\circ} 25.808$, Educación: estudiantes, embarazadas y progenitores. (28 de noviembre del 2003). Recuperado del sitio de internet de InfoLEG: http://servicios.infoleg. gob.ar/infolegInternet/verNorma.do?id=90598

Mancini, I. (2004). Modelos de maternidad entre las jóvenes de los sectores medios de Buenos Aires. Ponencia presentada en el Congreso Argentino de Antropología Social, 25-28 de mayo. Córdoba, Argentina.

Marcus, J. (2006). Ser madre en los sectores populares: una aproximación al sentido que las mujeres le otorgan a la maternidad. Revista Argentina de Sociología, 4(7), 100-119.

Margulis, M. (2008). Introducción. En M. Margulis (Ed.), La juventud es más que una palabra. Ensayos sobre cultura y juventud (pp. 9-12). Buenos Aires: Biblos.

Margulis, M., y Urresti, M. (1996). La juventud es más que una palabra. En M. Margulis (Ed.), La juventud es más que una palabra. Ensayos sobre cultura y juventud (pp. 13-30). Buenos Aires: Biblos.

Méndez, L. (1986). ¿La impertinencia de hablar de juventud rural? México D. F.: CREA.

Merello, A. (1973). Prospectiva. Teoría y práctica. Buenos Aires: Guadalupe.

Prensky, M. (2001). Digital natives, digital immigrants. On the Horizon, 9(5). Recuperado de https://www.marcprensky.com/writing/Prensky\%20-\%20Digital\%20Natives, \%20Digital\%20Immigrants\%20-\%20Part1.pdf

Reguillo, R. (2000). Estrategias del desencanto. Emergencias de culturas juveniles. Buenos Aires: Norma, colección Enciclopedia Latinoamericana de Sociocultura y Comunicación.

Relevamiento Unicef (2019). Escuelas secundarias rurales mediadas por TIC. Recuperado de https://www.unicef.org/argentina/escuelas-secundarias-rurales-mediadas -por-tic 
Reygadas, L. (2008). La apropiación. Destejiendo las redes de la desigualdad. México, D. F.: Anthropos.

Sen, A. (1999). Development as freedom. Nueva York: Alfred A. Knopf.

Sen, A. (2004). Nuevo examen de la desigualdad. Madrid: Alianza Editorial.

Van Dijk, J. (2005). The deepening divide. Inequality in the information society. Thousand Oaks, CA: Sage Publishing.

Vio Grossi, G. (1986). Juventud rural: ¿nuevos actores en el campo? Santiago de Chile: PIIE.

Vommaro, P. (2014). Juventudes, políticas y generaciones en América Latina: acercamientos teórico-conceptuales para su abordaje. En S. Alvarado y P. Vommaro, (Comps.), En busca de las condiciones juveniles latinoamericanas. Buenos Aires: CLACSO.

Vommaro, P. (2015). Movilizaciones juveniles en América Latina actual: hacia las configuraciones generacionales de la política. Controversias y Concurrencias Latinoamericanas. Recuperado de https://ri.conicet.gov.ar/bitstream/handle/113 36/54024/CONICET_Digital_Nro.fdb2dda2-8ae8-4f4b-95cb-699b393972e5_A. pdf?sequence $=2 \&$ isAllowed $=y$ 